\title{
Una aproximación compleja de forma y función del uso linguiístico y de teoría y práctica de la enseñanza de la gramática de ELE
}

\author{
A complex approach to form and function in linguistic usage and theory and practice \\ in grammar teching of Spanish as a foreign language (SFL) \\ Beatriz GRANDA* \\ Universidad Nacional Autónoma de México (UNAM)
}

RESUMEN: La enseñanza de la gramática continúa siendo motivo de debate, ya que tanto los contenidos como las metodologías utilizados en clase no han variado mucho: se prioriza, generalmente, la descomposición de sus partes constituyentes y los procedimientos de identificación de categorías (Bosque y Gallego, 2016). En este artículo se exponen las características de una materia de un programa de especialización (nivel posgrado), que parte del discurso como unidad de enseñanza; su objetivo es presentar la lengua desde una perspectiva compleja, con contenidos y procedimientos (teoría y práctica vinculadas) que capaciten al profesor de español como lengua extranjera (ELE) para:

- integrar los planos morfológicos, sintácticos, semánticos y discursivos en el análisis y explicación del funcionamiento de la lengua;

- vincular el uso de la morfología del pasado con categorías discursivas narrativas y con procedimientos referenciales que lleva a cabo el narrador en la situación de enunciación del discurso (Granda, 2014).

PALABRAS CLAVE: Gramática. Discurso. Forma-función. Enseñanza

ABSTRACT: Grammar teaching continues to be a debate issue, since contents as much as methodologies used in classrooms haven't varied too much: emphasis is put on the decomposition of units and on procedures for category identification (Bosque y Gallego, 2016). This article presens the characteristics of a graduate course (specialization level) that takes the discourse level as teaching unit. The purpose is to discuss the language from a complex

\footnotetext{
* Doctora en Lingüística. Universidad Nacional Autónoma de México. Centro de Enseñanza para Extranjeros. Ciudad de México. E:mail: bgranda@unam.mx
} 
perspective with contents and procedures (relating theory and practice) that allows Spanish as a Foreign Language (SFL) teachers to:

- integrate morphological, syntactic, semantic and discursive levels in the explanatory analysis of language functioning;

- Link the use of past tense morphology with narrative categories and referencial procedures used by the narrator in a discourse enunciative situation (Granda, 2014).

KEYWORDS: Grammar. Discourse. Form-function. Teaching.

\section{Introducción}

En este artículo se presenta el diseño pedagógico, entendido como el conjunto de teorías y modelos que subyacen a la toma de decisiones de los contenidos y procedimientos que conforman una asignatura para la formación de profesores de ELE. Esta materia se titula "Discurso y gramática: Tiempo y aspecto en la narración" y es parte del último semestre de un programa de especialización (nivel posgrado) del Centro de Enseñanza para Extranjeros de la Universidad Nacional Autónoma de México (UNAM). Tiene carácter optativo, por lo cual los estudiantes, maestros en formación o en ejercicio de la docencia que la seleccionan, suelen tener un interés particular en el tema que se aborda. Con frecuencia es el tema (o uno de los temas) de su trabajo final.

Al enfrentarse a la enseñanza de una lengua extranjera, lo que el docente necesita es explicar su funcionamiento, su significado en contextos situados de uso y su vinculación con los recursos lingüísticos disponibles para llevar a cabo esta tarea. Con ese propósito, en esta asignatura se ofrece al maestro en formación o actualización herramientas de análisis de la lengua que le permitan ir más allá de enfoques "formalistas", que prevalecen en la enseñanza y que, si bien son imprescindibles, resultan insuficientes para comprender la complejidad que involucra la comunicación lingüística. Se analiza particularmente el uso de la morfología verbal del pasado en el discurso narrativo. El discurso se concibe como el acto comunicativo, como "lo único observable de la lengua" (Caravedo, 2014); es el ámbito en el que se integra lo formal con lo funcional del uso lingüístico: los aspectos morfosintácticos, semánticos y pragmáticos involucrados en la comunicación lingüística. 
Por otro lado, se vincula este enfoque de análisis de la lengua con estudios sobre su adquisición, específicamente sobre el desarrollo de la morfología verbal del pasado en ELE y las implicaciones que se derivan para su enseñanza.

Los supuestos teóricos y su aplicación en el diseño de la asignatura se ejemplificarán con contenidos, actividades y ejercicios de las tres unidades que la conforman. Su objetivo general es que el estudiante reflexione sobre el uso de la morfología verbal de tiempo y aspecto del subsistema del pasado en español, con énfasis en las formas simples del pretérito y copretérito y su realización en situaciones de enunciación discursiva y aplique este conocimiento en propuestas didácticas para su enseñanza (Granda, 2008).

\section{Lo formal y lo funcional en la enseñanza de la lengua}

La enseñanza profesionalizante se ve como la necesidad de formar "expertos", lo que se entiende como poseedores de una colección de conocimientos teóricos sobre una materia en particular (Schön, 1998), que en el campo de la didáctica de lenguas se traduce fundamentalmente en el conocimiento del sistema modélico, el "estándar académico" (Moreno Fernández, 2010). Esta manera muy instaurada de asumir la enseñanza, que sigue vigente en gran parte de los programas de formación docente, proviene de una visión del objeto de estudio, la lengua, y particularmente de su gramática, como un sistema cerrado, completo, estable, que podemos fragmentar en unidades y niveles, cuyo estudio se enfoca principalmente a etiquetar las categorías lingüísticas y describirlas a manera de reglas de cómo combinar sus componentes, generalmente al nivel de la oración y con ejemplos prototípicos (Camps, 2010; Bosque y Gallego, 2016).

Se pretende luego que el docente aplique estos conocimientos, que se suponen garantizan la enseñanza de la lengua, en el contexto particular, diverso y siempre cambiante del salón de clases. En este ámbito, el maestro se ve en la necesidad de dar respuesta a situaciones emergentes, a explicaciones sobre el uso de la lengua, su

variación y las múltiples posibilidades que tiene el hablante en la selección de los recursos lingüísticos para funcionar en situaciones de comunicación genuina. 
Si bien son indudables los avances alcanzados en las últimas décadas que incorporan aspectos funcionales del uso de la lengua en su enseñanza, no se ha logrado todavía ofrecer una perspectiva integradora que dé cuenta de la relación entre el conocimiento sistemático y consciente de la gramática y el desarrollo de las habilidades discursivas de la lengua (Serrano, 2006).

En esta asignatura, se busca que el maestro-estudiante aborde el análisis de la lengua en el contexto del discurso, ámbito apropiado para estudiar la gramática, por ser no sólo el lugar donde la gramática se pone en uso, sino también la fuente a partir de la cual se forma o "surge" la gramática (Ochs, 2003, en Granda, 2005). Nos enfocamos al análisis del uso de la morfología verbal del pasado, particularmente del pretérito y copretérito, porque representa un aspecto complejo para su adquisición por parte de hablantes no nativos de la lengua. La expresión del "tiempo" en español forma parte de un "sistema altamente estructurado y especializado en la representación de un dominio cognitivo esencial en nuestra existencia" (Paris, 2007). Las lenguas naturales difieren ampliamente en cuanto a la manera de codificar el significado del "tiempo" (ver Comrie, 1989), por lo que la enseñanza de las particularidades de la lengua extranjera representa una tarea ardua para los docentes.

Cabe destacar que en el diseño de esta asignatura, procuramos vincular los conocimientos con los procedimientos para alcanzarlos, es decir asumir la teoría y la práctica como dos dimensiones que se retroalimentan (Schön, 1998).De allí que se otorgue preponderancia a una metodología dirigida a promover el análisis, la reflexión y la discusión de las posibilidades de uso de la lengua para expresar y relacionar eventos en el tiempo y buscar explicaciones en paradigmas que van más allá de los valores y significados que conforman el sistema estandarizado.

Se trata de una asignatura conformada por tres unidades: una primera unidad destinada a activar los conocimientos previos del maestro en formación sobre las principales categorías de análisis de la morfología verbal del pasado en español; una segunda, que conforma la propuesta central de la materia, toma el discurso narrativo como ámbito de realización de la lengua y se enfoca en contextos discursivos específicos vinculados con la expresión del "tiempo" y una tercera unidad, en la que se entroncan los temas de las dos primeras, con sus implicaciones en la adquisición, 
aprendizaje y enseñanza de la morfología verbal. Su diseño queda representado gráficamente como sigue:

Gráfica 1: Contenidos de las unidades de la asignatura

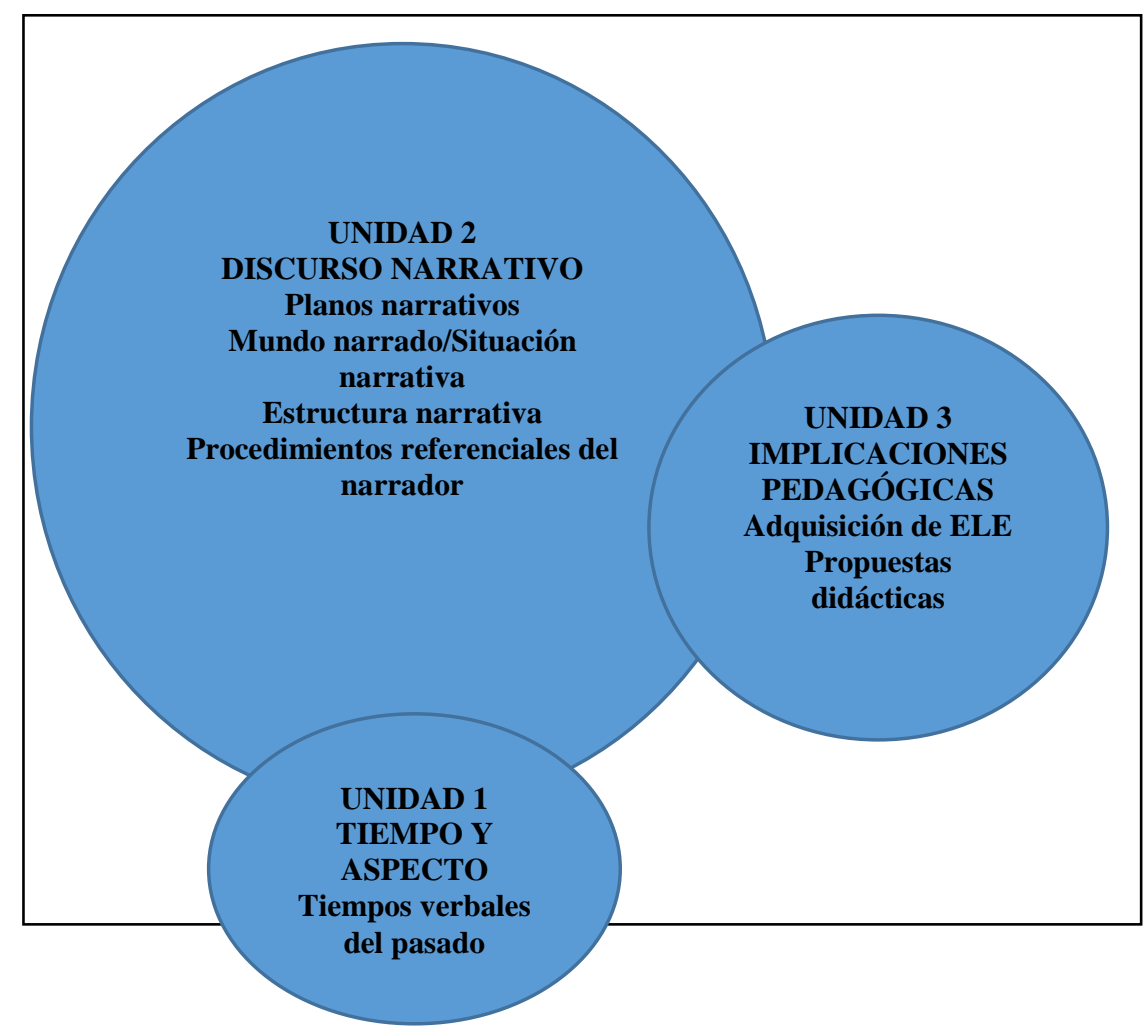

En los apartados que siguen, se expondrán los temas específicos de cada unidad con énfasis en ejemplos seleccionados de algunas actividades representativas ${ }^{1}$ de los procedimientos y contenidos involucrados en la metodología propuesta.

\section{Activación de conocimientos previos (Unidad 1)}

Incorporamos una primera unidad introductoria, en la que los maestros en formación o actualización retoman conocimientos adquiridos en materias previas del programa,

\footnotetext{
${ }^{1}$ Por razones de espacio, se seleccionaron 6 de 18 actividades que conforman la materia, sin embargo, son actividades que pueden ilustrar la propuesta de los procedimientos implicados para promover una relación entre lo formal y lo funcional, entre la teoría y la práctica, en la búsqueda de soluciones a los problemas que se presentan en la enseñanza.
} 
específicamente las que se refieren al TIEMPO VERBAL, al ASPECTO GRAMATICAL y al ASPECTO LÉXICO y utilizan estas categorías en el análisis y la reflexión de posibles selecciones que hace el hablante para expresar eventos en el tiempo. El objetivo principal es sensibilizar al estudiante sobre la insuficiencia de abordar la enseñanza con base en la aplicación de estas categorías en forma de reglas gramaticales y ejemplos modélicos a nivel de la oración para explicar el uso de la lengua. Con ese propósito usamos muestras de lengua tomadas de producciones genuinas de hablantes nativos, de textos literarios, de alumnos de ELE y también manipulamos o creamos ejemplos cuando se requiere para mostrar ciertos contrastes ${ }^{2}$.

En esta presentación de la materia, incluiremos solo definiciones y características $^{3}$ generales de las principales categorías que los estudiantes manejan y aplican en las actividades, ejercicios y procedimientos, representativos de la materia, que se diseñaron para el análisis y la reflexión (individual, en equipo y con mucha frecuencia a través de discusiones en foros grupales) de usos de la lengua.

Definimos el tiempo verbal como "una categoría gramatical deíctica mediante la cual se expresa la orientación de una situación bien con respecto al punto central (el origen) bien con respecto a una referencia secundaria que, a su vez, está directa o indirectamente orientada con respecto al origen" (GRAE, 1999:2879).

Desde esta perspectiva temporal, los tiempos de la morfología verbal pueden considerarse como "absolutos" o "relativos" (Bello, 1947, 1984)4: los absolutos tienen como referencia el momento del habla (presente, pretérito y futuro), mientras que las formas relativas tienen como referencia los tiempos absolutos, con respecto a los cuales pueden estar en relación de anterioridad (antecopretérito), simultaneidad (copretérito) o posterioridad (pospretérito). Las formas compuestas, que completan el subsistema del pasado en indicativo, tienen en común un significado de anterioridad respecto del tiempo del auxiliar: he amado es un antepresente; hube amado, antepretérito; habré amado antefuturo; había amado, antecopretérito y habría amado antepospretérito.

\footnotetext{
${ }^{2}$ Son "datos negativos", los cuales que resultan fundamentales para el lingüista porque le ayudan a establecer los límites combinatorios de las palabras (Bosque y Gutiérrez ,2009 p.49).

${ }^{3}$ En la asignatura se incluye una información más amplia, como así también ligas a los contenidos de materias previas (por tratarse de una materia en línea nos permite esa posibilidad), y una selección de documentos y referencias bibliográficas sobre el tema, al que pueden recurrir para ampliar la información. ${ }^{4}$ Incluimos la teoría de Bello por ser el punto de arranque de enfoques más actuales y por ser altamente productiva en el campo de la didáctica de ELE.
} 
Retomamos también la noción de punto de referencia temporal (R), que incluye más de un punto para orientar las situaciones dentro del sistema verbal ${ }^{5}$. Así, el PUNTO DEL EVENTO (E) denotado por el verbo en Chucho escribió una carta, sitúa el evento en el pasado y tiene su referencia directa con el MOMENTO DE HABLA $(\mathrm{H})$; mientras que en Chucho estaba cansado cuando escribió la carta, el E tiene como PUNTO DE REFERENCIA (R) a escribió, que a su vez es anterior a $\mathrm{H}$.

Por otro lado, definimos el concepto general de ASPECTO como "las diferentes formas de ver la constitución temporal interna de una situación” (Comrie, 1976:3), y consideraremos dos acercamientos a la cuestión del aspecto:

El ASPECTO GRAMATICAL es una categoría estrictamente formal que se enfoca en la distinción de la flexión verbal o los auxiliares, como en Chucho durmió bien anoche, cuyo E en pretérito denota su completitud; mientras que en Chucho dormía cuando sonó la alarma, el E, codificado en copretérito presenta la situación en una parte de su desarrollo, sin referencia a sus límites temporales (Colombo, 2015).

El ASPECTO LÉXICO es el o significado o contenido semántico inherente al predicado (verbo y sus argumentos). Distinguimos, de acuerdo con la clasificación de Vendler (1967), la teoría clásica más usada en los estudios sobre este tema, en:

REALIZACIONES (eventos dinámicos, durativos y télicos, como en Chucho escribió una carta.

LOGROS (dinámicos y puntuales), como en Chucho salió de la casa.

ACTIVIDADES (dinámicas y atélicas, como en Chucho canta bien las rancheras.

ESTADOS (situaciones estables, sin dinamismo) como en Chucho estaba muy divertido en la fiesta. ${ }^{6}$

Tomando estos conceptos como herramienta de análisis, se trabaja básicamente con actividades en las que los estudiantes tienen que explicar el significado de la morfología verbal en muestras de uso de la lengua. Particularmente en contextos de variación de esta morfología, que dependen de la perspectiva de la situación por parte del hablante y de las posibilidades combinatorias de las formas lingüísticas en el

\footnotetext{
${ }^{5}$ Se entiende por sistema verbal al conjunto estructurado de las formas que componen la conjugación.

${ }^{6}$ Se ofrecen pruebas sintácticas para la clasificación del aspecto léxico a través de una liga en la que se exponen los diferentes resultados que producen los predicados cuando la clase aspectual del verbo entra en contacto con determinadas expresiones temporales y tiempos gramaticales (Morimoto, Y, 1998).
} 
contexto sintáctico en que aparecen. Se busca que la reflexión que se promueve, le permita al estudiante tomar conciencia de la complejidad que involucra la expresión y relación de eventos en el tiempo donde, para la interpretación de su significado intervienen tanto el tiempo verbal como el aspecto gramatical codificado en la morfología, así como la variedad aspectual (aspecto léxico) de los verbos y, en algunos contextos, los complementos adverbiales.

Para el diseño de estas actividades se seleccionaron contextos de uso lingüístico, como los incluidos en la siguiente Actividad 1, en los que se muestra cierta variación en el uso de la morfología por parte del hablante, como en (1) y ( $\left.1^{\mathrm{a}}\right)$, donde se expresa un mismo o similar significado o cómo en (2) y (2a) en el que su uso está restringido a una sola forma, ya que su sustitución llevaría a un cambio de significado.

O bien, cómo el hablante puede expresar diferentes perspectivas de una misma situación similar al variar el uso de la morfología verbal en un mismo contexto como en (3) y (3a).

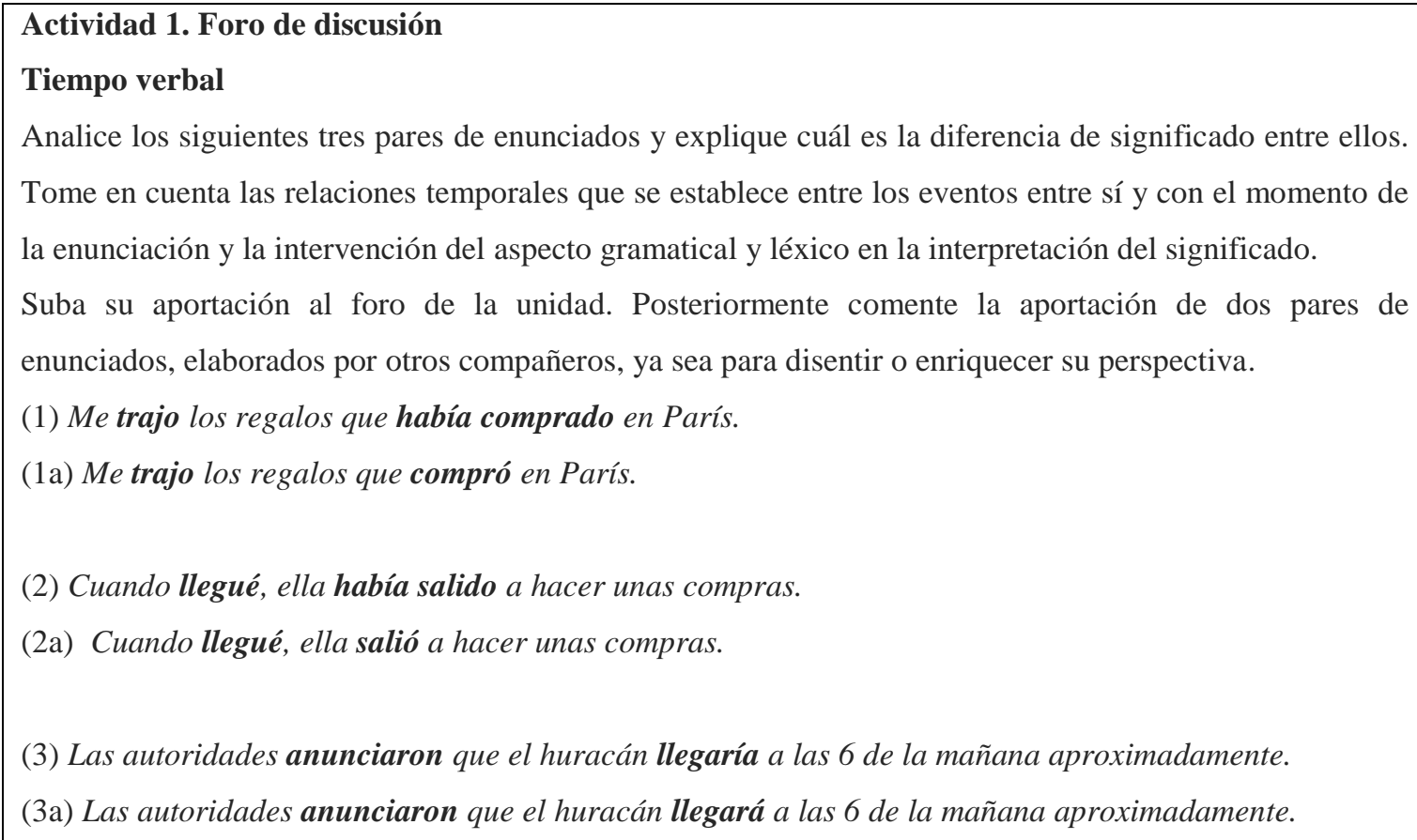
enunciados, elaborados por otros compañeros, ya sea para disentir o enriquecer su perspectiva.

(1) Me trajo los regalos que había comprado en París.

(1a) Me trajo los regalos que compró en París.

(2) Cuando llegué, ella había salido a hacer unas compras.

(2a) Cuando llegué, ella salió a hacer unas compras.

(3) Las autoridades anunciaron que el huracán llegaría a las 6 de la mañana aproximadamente.

(3a) Las autoridades anunciaron que el huracán llegará a las 6 de la mañana aproximadamente.

La discusión que genera una actividad como la anterior es muy amplia y diversa; en términos generales, de acuerdo con la reflexión que se busca promover, los aspectos con que más frecuencia se discuten son los siguientes:

En la oración (1), el hablante expresa anterioridad (había comprado) a un pretérito, que es su tiempo de referencia (R) (trajo), mientras que en (1a), el hablante 
puede sustituir el antecopretérito por el pretérito (compró) que, aunque su referencia directa es el Momento de $\mathrm{H}(\mathrm{H})$, su anterioridad con respecto a trajo se infiere por su uso en el contexto sintáctico en que aparece.

En (2), en cambio, no es posible la sustituir el tiempo verbal: había salido por salió en (2a) sin afectar el orden y la relación temporal que denotan los eventos: en (2) se expresa una relación de anterioridad entre los eventos con el uso del antecopretérito (había salido), cuya $\mathrm{R}$ es el pretérito y en (2a) se expresa una secuencia cronológica entre salió y llegué. En la interpretación de este último significado intervienen el tiempo verbal -dos pretéritos que tienen al $\mathrm{H}$ como su referencia directa-, el aspecto gramatical perfectivo codificado en ambos eventos, la variedad léxica de los verbos (ambos son cumplimientos) y el contexto sintáctico en el que aparecen: con el uso de cuando en un complemento adverbial oracional, combinado con dos eventos puntuales (cumplimientos) en pretérito se expresa una relación de secuencia entre la oración subordinada con la principal.

En (3) y (3a), los estudiantes comparan la posibilidad que tiene el hablante de expresar diferentes perspectivas de una situación similar dependiendo de la perspectiva que adopte. En (3), la relación que se establece en llegaría es de posterioridad, con referencia a anunciaron. Mientras que en (3a), el hablante escoge expresar posterioridad (llegará) con respecto al $\mathrm{H}$. La perspectiva del hablante en este último enunciado es expresar la posibilidad futura de la llegada del huracán en el presente enunciativo que comparten hablante e interlocutor.

Obsérvese el siguiente ejemplo de una actividad de foro, cuyo objetivo es concienciar al maestro en formación o actualización de la necesidad de incluir un contexto discursivo (más allá de la oración) para la interpretación de la distinción aspectual en la representación del significado de la morfología verbal usada.

\section{Actividad 2. Individual o en equipo.}

\section{Aspecto gramatical y léxico}

En equipos de dos estudiantes, o de manera individual, según su preferencia, analice los siguientes grupos de enunciados a partir de las categorías de aspecto gramatical y aspecto léxico y explique el significado de cada uno. Agregue un contexto más amplio en el que quede clara la distinción entre los enunciados (pueden hacer algunas modificaciones de los enunciados si lo cree necesario)

(1) Se sentó, pidió el menú y escogió un platillo. 
(1a) Se sentaba, pedía el menú y escogía un platillo.

(2) En el 2007, tuvo un hijo.

(2a) En el 2007, tenía un hijo.

Suban su aportación a la carpeta compartida. Escojan un grupo de enunciados perteneciente a otro compañero o equipo para disentir o enriquecer el análisis.

En la actividad anterior se analizan secuencias de eventos "completados" en pretérito (1) y secuencias habituales en copretérito (1a) en las que el significado de cada enunciado se deriva de la distinción perfectiva- imperfectiva de la morfología usada (pretérito y copretérito). Se reflexiona en cómo su comprensión queda clara en contextos discursivos más amplios, que los estudiantes aportan y sobre los que se discute entre los diferentes equipos.

Se reflexiona sobre la interrelación del aspecto gramatical y el aspecto léxico en la representación del significado en (2) y (2a). Particularmente, en este ejemplo, existe una interferencia de ambos aspectos que resulta ardua de adquirir, de acuerdo con estudios de adquisición (Granda y Salaberry, 2013). Obsérvese que el uso de un verbo estativo cambia su perfil temporal si se usa pretérito; lo que se señala con esta combinación, en algunos contextos, es el ingreso a un estado o un cambio de estado (Morimoto, 1998). Son formas (tenía y tuvo) que se aprenden en los niveles iniciales de ELE, para cuya distinción, en los libros de texto, se suelen usar adverbios o frases adverbiales que contribuyen a su significación (Baker y Quesada, 2009). Sin embargo, se hace notar al estudiante que si bien la inclusión de una frase adverbial puede ayudar a su comprensión en ciertos contextos, en otros, como en los ejemplos anteriores, no queda claro el significado. Los estudiantes proporcionan contextos más amplios en donde la distinción de ambas formas sea clara, como los siguientes:

(2) En el 2007, tuvo un hijo con su novio de toda la vida. Un par de meses después, se casaron.

(2a) En el 2007, cuando se casó, ya tenía un hijo de dos o tres años.

Estas actividades provocan discusión e intercambio de opiniones, consensos y disensos entre los estudiantes, que son dirimidos, en la mayoría de las situaciones y que 
le permiten al futuro docente tomar conciencia de la necesidad de incluir el contexto del discurso para la interpretación del significado de esta morfología verbal.

Por otro lado, con este tipo de actividades, se representan, de alguna manera, situaciones del aula de clase en las que el maestro necesita explicar el uso de las formas de la lengua meta, vinculadas con su significado y su funcionamiento en la comunicación. Se reproduce la actividad investigativa que lleva a cabo de manera constante el docente en la solución de los problemas de clase, específicamente al tener que dar explicaciones sobre el uso de la lengua en discursos concretos.

La inclusión del discurso, entendido como ámbito de realización de la lengua, para la interpretación del significado de la compleja morfología verbal del pasado en español que se propone en esta materia se aborda en la siguiente unidad 2 de la materia.

\section{EI discurso como ámbito de enseñanza de la lengua (Unidad 2)}

Esta unidad (la medular del programa) se enfoca en el tipo de conocimiento sobre la lengua que demanda la tarea de vincularla con su significado en la comunicación; saberes sobre el objeto de enseñanza, que involucran las posibilidades de articulación de las formas del sistema para expresar funciones comunicativas. Se toman los datos que provienen del código y luego se vinculan con elementos extralingüísticos que condicionan el uso del lenguaje.

Para la interpretación del significado de la morfología del pasado se combina el componente formal con un componente discursivo, enunciativo y pragmático; particularmente se toman en cuenta recursos formales, "potenciales" (Halliday, 1979) que utiliza el hablante para dar prominencia a ciertos eventos y para relacionarlos en el tiempo de la narración, a partir de procedimientos referenciales que lleva a cabo para expresar su propia perspectiva temporal de las situaciones del relato (Granda, 2014).

En el diseño de las tareas y actividades de esta unidad se ofrece al maestro una metodología de enseñanza-aprendizaje, con vistas a ser usada como procedimiento de análisis y como herramienta explicativa del uso de la morfología verbal en el aula de clases.

Con ese propósito se incluyen fragmentos discursivos que tienen un sentido completo y cumplen una función comunicativa (micro o macrofunciones); son unidades 
oracionales, transoracionales o discursivas (vinculadas con la macroestructura del modo discursivo).

Se incluye también información y actividades destinadas a identificar y analizar y crear fragmentos discursivos que constituyen ámbitos temporales aspectuales con funciones específicas, que son: los PLANOS NARRATIVOS (PN): PRIMER PLANO (PP) y PLANO DE FONDO (PF) y el MUNDO NARRADO (MN) y la SITUACIÓN NARRATIVA (SN).

El contraste entre PN proviene de una distinción ampliamente analizada en los estudios de narrativa: el contraste entre el primer plano y el plano de fondo. Algunos estudios (ver Granda, 2005) sugieren que este contraste en planos es universal y que tiene sus orígenes en funciones cognitivas, comunicativas y posiblemente psicológicas, por lo tanto se puede esperar que esta distinción se manifieste, de alguna manera, en el discurso narrativo de la mayoría, sino de todas las lenguas.

El PP está constituido por eventos, al menos dos eventos que llevan la línea principal de la historia, típicamente conformada por verbos de acción, aspectualmente perfectivos; por otro lado, el material de soporte del PF está típicamente codificado en la forma de predicados estativos o durativos, aspectualmente imperfectivos. En español, son el pretérito y el copretérito las formas que cumplen esta función contrastiva de la organización del relato (Colombo, 2015). El pretérito contribuye a dar relieve a PP, enfocando los eventos que permiten la progresión de la narración y el imperfecto copretérito- describe las circunstancias, características, costumbres, condiciones, explicaciones, etcétera, como en: El primer día en la casa de la playa, me desperté y escuché el ruido de un motor. El cuarto estaba lleno de luz, pero al mirar el reloj vi que todavía no eran las seis de la mañana", en donde lo marcado "en negritas" expresa las funciones del PP narrativo y lo subrayado el PF.

Por otro lado, además del uso del copretérito para expresar el PF, este ámbito es más complejo en términos de los recursos gramaticales temporales que lo pueden constituir y las funciones específicas que expresan, ya sea (Bardovi- Harlig 2000: 282) revelando un evento anterior, localizando antes de los eventos del PP, como en Escuchó las noticias hasta que se percató que había pasado un largo rato, o haciendo una

\footnotetext{
${ }^{7}$ Fragmento de texto tomado de La casa de la playa de García Ponce, Juan.
} 
predicción de un evento por venir, posterior a los eventos de la secuencia narrativa del PP, como en Después de esta prueba, supe que si podría vivir en la Ciudad de México.

El MN y la SN se definen con base en los procedimientos referenciales que lleva a cabo el hablante en la producción de narraciones. La narración es parte de una situación de enunciación comunicativa; no existe un texto o discurso fuera de la realidad de las prácticas comunicativas concretas. El modo narrativo, así considerado, en su condición de producción involucra dos ámbitos bien identificados: el $\mathrm{MN}$, en que los personajes, sucesos y lugares de la historia se interrelacionan en un contexto temporal que lo individualiza y lo circunscribe (Schiffrin, 1994, en Granda, 2007), que tiene generalmente al pretérito del PP como referencia temporal se y la $\mathrm{SN}$, ámbito del presente de la enunciación narrativa. El narrador, al producir un relato, puede hacer referencia a uno u otro ámbito, para dar cuenta de los sucesos que conforman la trama de la historia (MN), como de los comentarios o evaluaciones desde el "aquí" y "ahora" del narrador (Granda, 2014). Estos ámbitos se ilustran en:

Quienes hayan leído con atención el relato de mis trabajos recordarán que un hombre de la tribu me siguió como un perro podría seguirme, hasta la sombra irregular de los muros. Cuando sali del último sótano, lo encontré en la boca de la caverna, estaba tirado en la arena, donde trazaba torpemente y borraba una hilera de signos, que eran como las letras de los sueños, que uno está a punto de entender y luego se juntan. ${ }^{8}$

En el texto anterior se presentan en "negritas" las formas características del MN con preponderancia de pretérito y copretérito para la expresión de los planos narrativos. Por otro lado encontramos eventos (subrayados) que están fuera del MN, en los que el hablante hace un alto en el relato de la trama narrativa y se dirige a su interlocutor para hacer un comentario o expresar situaciones vigentes en el momento de la enunciación o SN.

Como parte del contenido, se incluye información sobre los elementos de la estructura narrativa de Labov (1972) ${ }^{9}$ para mostrar la tendencia bastante generalizada (Colombo, 2015) del uso de pretérito del español como forma verbal que expresa el

\footnotetext{
${ }^{8}$ Fragmento de texto tomado de El Aleph de Borges, J.L.

${ }^{9}$ Las narraciones que están "más plenamente conformadas exhiben los siguientes rasgos estructurales" (Labov, 1972: 363): Resumen, orientación, complicación de la acción, evaluación (interna y externa) resultado o resolución y epílogo o coda.
} 
"resumen" (Esta es la historia de mi abuelo, que fue asesinado porque fue un juez justo). Asimismo, en pretérito se encuentran las oraciones propiamente narrativas, que llevan la secuencia de los hechos narrados, y la "resolución", con la cual se da por concluido el relato (equivalente al PP). También podemos encontrar usos del pretérito en evaluaciones o comentarios "incrustados" en la trama narrativa que no forman parte de su secuencia (Cuando llegué al lugar del accidente, vi que mi amigo ya había muerto. Fue la peor experiencia que tuve en mi vida) que expresa una evaluación con referencia al momento de la enunciación.

El copretérito aparece como la forma típica de la sección de orientación. En ella se describen las circunstancias que permiten introducir y ambientar la futura trama del relato (Mi abuela siempre me contaba historias de cuando yo era niña, me decía que...). A partir de esta información (que presentamos de manera resumida por cuestiones de espacio, pero que se encuentra suficientemente desarrollada en los contenidos de la unidad), se promueve la reflexión del uso de los recursos lingüísticos en nuevos paradigmas de análisis y el contraste de esta perspectiva con los planteamientos formales. Se utilizan con ese propósito actividades y tareas como las que se ilustran a continuación.

\section{Actividad 3. Trabajo individual \\ Planos narrativos}

Observe el cortometraje "El héroe" (https://www.youtube.com/watch?v=CnWxYImN4nQ) y realice las siguientes actividades:

- Escriba una breve introducción para “orientar” sobre el personaje principal y sus circunstancias:

(1) ¿Quién era Juan? ¿Dónde vivía? ¿Qué hacía?

- Complete los siguientes enunciados con secuencias de acciones (PP):

(2) A las 8 de la mañana, Juan entró al metro, bajó las escaleras y...

(3) El policía lo tomó del brazo y lo arrastró fuera de la estación,.....

- Complete los siguientes enunciados con una descripción del escenario (PF):

(4) Cuando entró al andén, había mucha gente...

(5) En ese momento vio una muchacha parada junto a la línea amarilla,...

Posteriormente analice el tiempo verbal y el aspecto gramatical de la morfología usada y su combinación con el aspecto léxico de los verbos. 
Suba su archivo a la carpeta compartida.

\section{Actividad 4. Trabajo en equipo}

En equipo de tres estudiantes, identifiquen con dos colores distintos, el "mundo narrado" y la "situación narrativa" en los siguientes textos y envíenlos a la carpeta compartida.

Después, cada grupo evaluará el trabajo de otro equipo (que le indique el tutor) y subirá su evaluación a la carpeta compartida.

(1) Aquel día de espaldas sobre el tejado, escondido de mi padre, en silencio y absorto, observé a un gavilán en vuelo que luego de la rapiña regresaba a su nido con el pico ensangrentado. Nunca lo olvidé. ¿Cuál es la diferencia entre la vida y un instante? Hay un tiempo en que se cree que no hay otros límites que los escogidos por uno mismo. Pero al cabo comprendemos que todo puede suceder de pronto y para siempre en el andén de una estación, entre un tren y otro o al atardecer, en la fonda de un pueblo perdido y polvoriento. ${ }^{10}$

(2) En una ocasión que estábamos en Tecolutla, mi hermana y yo comenzamos a caminar a lo largo de la playa para recoger conchas. Era muy temprano y no había mucha gente nadando, ni asoleándose, aunque recuerdo que hacía mucho calor.

(3) Llegué a mi fiesta de graduación y me encontré con mi amiga Ketura, que fue mi mejor amiga en la escuela secundaria, ella estaba con su novio y me invitaron a sentarme con ellos.

(4) Un señor muy atento me indicó con todo detalle cómo llegar al Metro Chapultepec y qué camino tomar para la Universidad. $\quad$... Y eso hice. Llegué a la estación, subi escaleras, observé que hacían las personas, compré mi boleto y...

(5) Nos encontramos en el camino y me propuso que continuáramos juntos. Dudé un poquito pero luego acepté, su actitud me dio confianza.

En el ejercicio anterior, el estudiante generalmente analiza la distinción entre MN y SN, a partir de la identificación del PP y el PF (MN) y otras situaciones que están fuera de este ámbito (SN), cuya referencia directa es el H.

\footnotetext{
10 Tomado de Tizón, H. La mujer de Strasser
} 
En (1) hay un solo evento (observé) alrededor del cual se hace una reflexión. No es un texto narrativo, por lo tanto no podemos identificar el PP que constituye la referencia temporal del MN. En 2, hay algunas referencias al presente enunciativo $(\mathrm{H})$, como en aunque recuerdo. Son situaciones en las que el hablante hace un alto en el relato para dirigirse a su interlocutor y expresar situaciones vigentes en la SN.

En (3), el hablante se sale de la secuencia cronológica de los eventos narrados y usa el pretérito en fue mi mejor amiga en la escuela secundaria, para expresar una evaluación externa al relato y en (4), para expresar un resumen: $Y$ eso hice, en el que el hablante se anticipa a la secuencia de eventos que expresa más adelante.

En (5), encontramos clausulas narrativas (PP/MN) con excepción de su actitud me dio confianza, que es una clausula explicativa (lo cual se infiere por el sentido del fragmento); es decir no sigue la secuencia de los eventos. Los estudiantes reflexionan, en contextos, como este, de las inferencias pragmáticas que contribuyen a la interpretación del significado.

En una actividad como la anterior suele haber una diversidad de análisis y algunos disensos en la manera de explicar los usos. Cuando esto sucede, es una oportunidad para abrir un foro de discusión, en el que el tutor hace una síntesis de los acuerdos y desacuerdos para ser discutidos de manera grupal.

Cabe mencionar que los productos que surgen de las reflexiones y discusiones entre los estudiantes varían en cada aplicación de la materia. Los mismos estudiantes presentan perspectivas explicativas y ejemplos que el tutor puede usarlos en otras imparticiones de la materia o que sirven de base para proponer otras actividades de análisis.

\section{Implicaciones en la didáctica de ELE (Unidad 3)}

El objetivo de esta última unidad es mostrar la relación entre lo aprendido en las dos unidades previas con los resultados de estudios de adquisición de la morfología verbal en ELE, con vistas a que el maestro pueda usar este conocimiento en propuestas didácticas concretas.

Se proporciona información de los resultados de investigaciones sobre la adquisición de las formas del pasado en ELE, de manera que el maestro pueda diseñar 
material didáctico que facilite y acompañe (en términos de la secuencia de enseñanza) el proceso natural de adquisición de la morfología verbal (Blyth, 2005) en contextos del discurso. Por otro lado, se incluyen ligas a documentos ${ }^{11}$ con ejemplos concretos de lecciones y materiales didácticos que los estudiantes podrán analizar y tomar como base de nuevas propuestas de enseñanza.

La adquisición de la morfología verbal del pasado en español y en otras lenguas es un área central de investigación, dada la complejidad de los sistemas temporales aspectuales de las lenguas. Una de las hipótesis más aceptadas y probadas en diversas investigaciones es la llamada HIPÓTESIS DEL ASPECTO (HA) que muestra, en términos generales (para mayor información sobre estudios sobre esta hipótesis, véase en Ayoun y Salaberry, 2005), los siguientes resultados:

Los aprendices usan primero la marca perfectiva con logros o cumplimientos ("achievements") y realizaciones ("accomplishments") y luego se extiende gradualmente a actividades y estados. El pasado imperfectivo aparece después del pasado perfectivo, se restringe primero a estados y actividades y después se usa con realizaciones y logros. La adquisición de la marca progresiva inicialmente se restringe a actividades y luego se extiende a realizaciones y logros.

Los resultados de otras investigaciones, sugieren que en las primeras etapas de adquisición español es el pretérito la única forma usada independientemente de la categoría aspectual léxica del verbo. Cuando los hablantes tienen más experiencia en la L2, el uso de la marca de pasado será afectado por la semántica léxica del predicado; en Salaberry (2005:190) se exponen hallazgos empíricos provenientes de cuatro estudios en español como L2 (Liskin Gasparro, 2000; Lubbers-Quesada, 1999; Schell, 2000; Granda, 2004, citados en Salaberry 2005: 190) que son relevantes para esta hipótesis, según este autor.

Otra hipótesis, denominada HIPOTESIS DEL DISCURSO (HD) sugiere que existe una relación entre la organización en planos del discurso narrativo y el aspecto léxico y predice que la morfología verbal va a emerger en el interlenguaje de los aprendices para distinguir el PP del PF en el discurso narrativo. Los datos muestran que primero emergen los cumplimientos y las realizaciones, que son más frecuentes en el

11 Se describen posteriormente y se registran en la bibliografía de este artículo. 
PP. Las actividades se usan en copretérito, pero más frecuentemente en progresivo para el PF (Bardovi-Harlig, 2000).

En un estudio con aprendices anglohablantes, las mayores dificultades en el interlenguaje de los alumnos se evidencia en el uso de verbos de estado para la distinción de los planos narrativos; se bien son más frecuentes los verbos estativos para marcar el PF, persiste el uso del pretérito con estados en estos contextos (Granda y Salaberry, 2013).

La siguiente actividad está planteada para que el estudiante examine textos producidos por alumnos de ELE y los clasifique de acuerdo con la información sobre la secuencia de adquisición proporcionada por los estudios empíricos.

\section{Actividad 5. Trabajo individual \\ Nivel de desarrollo}

Ordene los siguientes textos de acuerdo con el nivel de desarrollo en la adquisición de las formas del pasado en: principiante, intermedio y avanzado. Explique las razones de su selección. Elabore su actividad en un editor de textos y súbalo a la carpeta.

(1) Llegó a la estación, bajó las escaleras y entró al andén. *Hubieron muchas personas. Muchos viejos y también jóvenes. Un niño que lloraba y estaba con su mamá. Juan se paró junto a otras personas...

(2) Muchos años pasado, un niño de cabrero se llama Juan. El es bromista. El habla "Los lobos llegan" para recurso. Pero el mente más que habla. Después los amigos no gustan Juan. Después el enfronta a lobos y los amigos no ayudamos para él.

"No mentir a diario"

(3) El guía les explicó que al menos deberían usar unas señales para indicar el camino porque en ese tiempo no tuvieron el mapa de la cueva...

(4) Se dio cuenta que el tren entraba a la estación y tenía un presentimiento, entonces avanzó hacia la chica que estaba parada tambaleándose después de la linea amarilla...

(5) El terapeuta los ayudó a ponerse de acuerdo, su opinión era decisiva para lograr ese arreglo.

(6) Un día fuimos al museo con mis amigos, había una exposición de Salvador Dalí. Fue muy interesante lo que pasó. Durante la visita, una amiga le mostró un extinguidor a otra amiga y le dijo que le gustaba mucho esa obra de Dalí. Era una broma, pero nuestra amiga no se dio cuenta y nos reímos muchísimo cuando dijo que a ella también le gustaba.

El ejercicio anterior está diseñado para que se analicen, principalmente, usos de verbos de estado como *hubieron (hubo) en lugar de había para expresar el PF en (1). Un texto que corresponde a un nivel principiante de acuerdo con el análisis de las 
formas usadas en el fragmento completo. El mismo error lo encontramos en el texto 3, en tuvieron en lugar de tenían; si bien este fragmento corresponde a un nivel intermedio, puede ser, al igual que (1), una muestra del uso del pretérito como forma única, que se extiende de niveles principiantes a niveles intermedios.

En (4) y (5), en cambio, se usa copretérito en lugar de pretérito. En 4, el uso adecuado de tuvo en lugar de copretérito, en ese contexto, corresponde a la expresión de un cambio de estado, que está en la línea de los eventos que llevan la secuencia narrativa. En (5), el uso adecuado de fue, en lugar de era, expresa una evaluación sobre un evento que efectivamente sucedió (el acuerdo logrado), evaluación que proviene de una perspectiva del hablante fuera de los eventos del MN. Ambos fragmentos se clasifican como correspondiente a un nivel avanzado y confirma la HA respecto de la adquisición tardía de pretérito con estados.

En el fragmento (2) se confirma lo que sostiene la $\mathrm{HD}$, en cuanto a la adquisición temprana del PP de la narración. Se trata claramente de un hablante de ELE principiante, que a falta del conocimiento de la morfología del pasado, usa complementos adverbiales para señalar tanto la localización de los eventos (Muchos años pasado) como la secuencia del PP (después, después) y por el orden en que va incorporando los eventos de acuerdo con la cronología de los acontecimientos.

El texto (6) muestra el uso adecuado de la distinción entre dos eventos en pretérito fuera del ámbito del MN (fue muy interesante lo que pasó) de una situación en copretérito que describe las circunstancias al interior del ámbito del $\mathrm{MN}$ (era una broma) de un estudiante avanzado de ELE.

Por último, se proporcionan ligas ${ }^{12}$ a dos artículos con propuestas didácticas que vinculan la perspectiva discursiva de abordaje de la morfología verbal del pasado con los estudios sobre su adquisición en ELE. Su objetivo general es que los alumnos analicen las propuestas y evalúen, con base en lo aprendido, las diversas actividades y tareas que se sugieren para la enseñanza del pasado en un foro de discusión.

Artículo 1: Se propone una diversidad de ejercicios para ser enseñados de acuerdo con el nivel de dominio del estudiante con respecto a los parámetros del MCER (Soulé y Granda, 2015)

\footnotetext{
${ }^{12}$ Su referencia se encuentra en la bibliografía del artículo.
} 
Artículo 2: Se propone una tipología de textos narrativos para la enseñanza de ELE, relacionada con la secuencia de adquisición de las formas del pasado, de acuerdo con investigaciones sobre el tema (Granda, 2008).

Después de esta actividad, en la que se evalúan y se enriquecen las propuestas de enseñanza con la participación del grupo, los alumnos deben hacer su propia propuesta como actividad final de la asignatura

\section{Actividad 6. Trabajo en grupo de dos o tres estudiantes}

\section{Propuesta didáctica}

Elaboren una unidad didáctica para enseñar la distinción de pretérito y copretérito en contextos discursivos específicos, incluyan explicaciones sobre el uso de la morfología verbal y al menos 6 actividades o tareas. Indiquen el nivel al que va dirigida.

Suban su propuesta a la carpeta compartida.

\section{Consideraciones finales}

Las tres unidades descritas previamente, responden a un diseño pedagógico en el que se integran y complementan tres dimensiones involucradas en la enseñanza de la gramática: las formas de la lengua a partir de su caracterización estructural como parte del sistema estandarizado (Unidad 1), su realización en el discurso (Unidad 2) y su importancia e implicaciones en la adquisición, y la enseñanza aprendizaje en el aula de ELE.

Así conformada la materia, el maestro en formación o actualización puede abordar el análisis de la lengua desde una perspectiva compleja y dinámica, con contenidos y procedimientos que lo capacitan para contrastar un enfoque "formalista" de enseñanza con un paradigma discursivo, más abarcador y más complejo en cuanto a las posibilidades del hablante de expresar nociones y relaciones temporales al interior de los relatos. Los recursos potenciales de la lengua sistémica, con que cuenta el hablante, para la producción del discurso se sistematizan, de manera más flexible, más abierta. Se ven como posibles realizaciones, no arbitrarias, sino motivadas por la misma lengua, (Serrano, 2006), en contextos narrativos específicos en los que el hablante puede otorgar prominencia a ciertos eventos (distinción de PN) y relacionar temporalmente los 
eventos del relato, de acuerdo con su particular perspectiva de las situaciones (distinción MN y SN).

Además, el diseño de las actividades y tareas enfocadas en procedimientos reflexivos y analíticos del uso, siempre variable de la lengua, promueve la actividad investigativa (que es parte del quehacer cotidiano del docente). No se pretende incidir en la docencia desde una teoría en particular, sino que, a partir de la búsqueda de soluciones explicativas que se requieren en el salón de clases, se recurre a la teoría, en busca de respuestas a la problemática del aula. Vemos esta relacional bidireccional entre teoría y práctica como requisito de un auténtico marco de construcción del conocimiento profesional docente.

Por último, la comprensión, por parte del docente, de la influencia conjunta de la clase aspectual con la estructura narrativa en el desarrollo del interlenguaje, le ofrece pistas para el diseño de materiales y secuencias que acompañen el proceso natural de adquisición de las formas del pasado en español.

De esta manera, las tres unidades de esta asignatura se vinculan y complementan desde una perspectiva formal y funcional de la lengua y con procedimientos analíticos que incorporan teoría y práctica de manera conjunta. Lo anterior responde a un diseño pedagógico que pone de relieve la actividad investigativa del docente.

\section{REFERENCIAS}

AYOUN, D. y R. SALABERRY. The development of L2 tense-aspect in the Romance languages. En AYOUN D. Y R. SALABERRY. Tense and Aspect in Romance languages. Philadelphia, Johnn Benjaming Publishing Company, 2005, p.1-34.

BAKER, J. y M. QUESADA. The effect of Temporal Adverbials in Selection of Preterit and Imperfect by Learners of Spanish L2. En: SELECT PROCEEDING OF THE 2009 SECOND LANGUAGE RESEARCH FORUM. Somerville MA, Cascadilla Proceeding Project, 2009, p. 1-15.

BARDOVI-HARLIG, K. Tense and Aspect. Language Learning, Vol. 50, 2000.

BLYTH, C. From empirical findings to the teaching of aspectual distinctions. En: AYOUN Y SALABERRY. Tense and Aspect in Romance Languages. Philadelphia, Johnn Benjaming Publishing Company, 2005, p. 211-252. 
BOSQUE I. Y A. GALLEGO. La aplicación de la gramática en el aula. Recursos didácticos clásicos y modernos para la enseñanza de la gramática. Revista de Lingüistica Teórica y Aplicada 54(2) II, 2016, p. 63-83.

BOSQUE, I. y GUTIÉRREZ R. Fundamentos de Sintaxis Formal. México, Siglo XXI, 2009.

CAMPS, A. Hablar y reflexionar sobre la lengua: hacia un modelo de enseñanza de la gramática basado en la actividad reflexiva en colaboración. En RIBAS SEIX (coord.) Libros de texto y enseñanza de la gramática. Barcelona, Ed. Graó, 2010, p.13-29.

CARAVEDO, R. Percepción y variación lingüística: un enfoque sociocognitivo. Frankfurt y Madrid, Vervuert/Iberoamericana, 2014.

COLOMBO, F. El subsistema de los tiempos pasado de indicativo en español: Semántica y sintaxis. México, UNAM, 2015.

COMRIE, B. Language universals and linguistic typology: Syntax and morphology. Cambridge, University Press, 1989.

GRANDA, B. Categorías narrativas y procedimientos referenciales aplicados a la didáctica de ELE. Revista Verbum et Lingua, Num. 3. Guadalajara, México, Universidad de Guadalajara, 2014, p. 22-35.

GRANDA, B. La enseñanza del pasado con un enfoque discursivo. Revista Decires. Volumen 11, Número 12-13. Centro de Enseñanza para Extranjeros, 2008. Disponible en: www.revistadecires.cepe.unam.mx/articulos/art12-2.pdf. Acceso en: 12 jun. 2018

GRANDA, B. Adquisición de tiempo y aspecto en textos narrativos en español como segunda lengua. En AKERBERG, M. Adquisición de segundas lenguas. Estudios y perspectivas. Colección: Líneas de Investigación en el Departamento de Linguística Aplicada. Centro de Enseñanza de Lenguas Extranjeras, UNAM, 2005, p. 87-110.

GRANDA, B. y R. SALABERRY. Funciones narrativas y verbos de estado en español como lengua extranjera. Revista Estudios de Lingüística Aplicada, $\mathrm{N}^{\circ}$ 57, Centro de Lenguas Extranjeras, UNAM, 2013, p. 51-71.

HALlidAY, M. El lenguaje como semiótica social. México, Fondo de Cultura Económica, 1979.

LABOV, W. Lauguage in the inner city: Studies in the black English vernacular. Philadelphia, University of Pensilvania, 1972. 
MORENO FERNÁNDEZ, F. Las variedades de la lengua española y su enseñanza. Madrid, Arco libros S.L, 2010.

MORIMOTO, Y. El Aspecto léxico: Delimitación. Madrid, ArcoLibros, 1998.

PARIS, L. Eventos e intervalos en la semántica del Pretérito, Imperfecto y Progresivo. Revista Signos 40 (65), 2007. p. 609-632

REAL ACADEMIA ESPAÑOLA. Nueva gramática de la lengua española. Madrid, Espasa Libros, 2009.

REINHART, T. Principles of Gestalt perception in the temporal organization of narrative texts. Lingüistics 22, 1984, p.779-809.

SALABERRY, R. Evidence of transfer of knowledge of aspect from L32 Spanish to L3 Portuguese. En AYOUN Y SALABERRY (eds.) Tense and Aspect in Romance languages. Philadelphia, John Benjamin, 2005, p. 179-210.

SCHÖN, D. El profesional reflexivo. Cómo piensan los profesionales cuando actúan. Ediciones Paidós Iberica S.A., 1998.

SERRANO, M. Gramática del discurso. Madrid, Ediciones Akal, 2006.

SOULÉ, M. y B. GRANDA. ¿Qué gramática aplicar para la enseñanza de la distinción imperfecto e indefinido? En VII JORNADAS DE FORMACIÓN PARA PROFESORES DE ESPAÑOL. Centro de Lenguas de la Universidad de Chipre, 2015. Disponible en: https://dialnet.unirioja.es/servlet/articulo? codigo=5338092. Acceso en: 7 jun. 2109. 